\title{
Extremely Large telescopes
}

\section{Jason Spyromilio ${ }^{1}$}

European Southern Observatory

Karl-Schwarzschild-Str 2, Garching bei Muenchen, D-85748, Germany

E-mail: jspyromieeso.org

Extremely large telescopes are being planned to address a number of the most profound questions in astronomy today. From the detection and study of extra-solar planets to the study of the first galaxies these optical and infrared telescopes are designed to provide powerful new probes in the field. In this paper, the current status and technical development of the European ELT project is described.

From planets to dark energy: the modern radio universe University of Manchester, Manchester, UK

1-5 October, 2007

Speaker 


\section{Introduction}

Extremely large telescopes are optical infrared reflectors with filled apertures of diameters of between 25 and 50-m. Since the 1990s with the operation of the Keck 10-m telescopes and the 8-m unit telescopes of the VLT as well as the Subaru, Gemini and Magellan telescopes there has been a clear scientific requirement to push to larger apertures and higher resolution. The maturity of adaptive optics systems enables a next generation of telescopes to be built whose resolution is no longer a priori limited by the earth's atmosphere. The scientific power of the telescopes can now be considered to increase as the diameter to the fourth power.

The term Extremely Large Telescope (ELT) was coined by Tom Sebring referring to a proposed successor to the 11-m Hobby Eberly Telescope in Texas. In a seminal paper presented at the 1996 Landskrona meeting Arneberg et al. described a 25-m "live" telescope design. By 1998 with the presentation by Gilmozzi of a 100-m telescope at the Kona SPIE meeting it was clear that the limit for telescope size was no longer technological. In the United States the decadal review placed the start of the construction of a 30-m telescope as the highest scientific priority items for the current decade. In the intervening years a number of designs for telescopes have been launched by the community. The California extremely large telescope, the Canadian very large optical telescope, the giant segmented mirror telescope, the Japanese Extremely large telescope, the Euro50, the 100-m OWL, the giant Magellan telescope and others. Following a number of years of independent development and much diversity of opinion in the most effective way to address the scientific goals, in the past two years a consolidation of the activities has led to three technically different and scientifically complementary projects. The thirty meter telescope (TMT) is a collaboration between Caltech, University of California and ACURA (Canada). The giant Magellan telescope with seven 8.4-m mirrors forming its primary is a collaboration between Carnegie, Harvard, the Smithsonian, MIT, Arizona, Texas at Austin \& Texas A\&M, Michigan and the ANU in Australia. In Europe following the OWL review at the end of 2005, an intense period of community consultation and collaboration in early 2006 concluded at the Marseille meeting with the community in November 2006 endorsing the design of a 42-m telescope. In December 2006, the ESO Council launched a three year phase B design study to result in a construction proposal to be presented to the ESO Council in late 2009.

The EELT PI is Roberto Gilmozzi and he is assisted by a Science Working group chaired by Marjin Franx \& Isobel Hook. The project scientist is Guy Monnet. Three project offices below the PI follow up the telescope, instrumentation and operations design activities. The author of this paper heads the telescope project office while Sandro D'Odorico and Fernando Comeron head the instrumentation and operations activities respectively. Generic design activities have been undertaken with the framework of an FP6 supported study. The bulk of the design funds however, 57.2 million Euro, have been allocated by the ESO Council directly out of the ESO budget. Within the FP7 activities some funds have been allocated to provide for further support of the Science Working Group activities and other programmatic matters. 


\section{Science cases \& Design reference missions}

The science cases for the three extremely large telescopes can be found in [1], [2] \& [3]. It is not only beyond the competence of the author to present or review them but also beyond the scope of this paper. It is however clear from the references above that almost no field of observational astronomy will be unaffected by the arrival of these telescopes.

In the European arena, multiple meetings with the community have produced a series of prominent science cases that are being used by the European ELT (EELT) science working group (SWG) to elaborate design reference missions (DRM). The DRMs are being elaborated at this time by the science working group and will be used to benchmark performance against the telescope and instrumentation design choices.

The first three prominent science case DRMs are in the areas of planet detection, resolved stellar populations and galaxy formation at high redshifts. Another 11 design reference missions will be developed in the forthcoming months. It is clear that not only is there enthusiastic support for the project in the community but also a heavy oversubscription of all the telescopes can be safely assumed.

The ELTs shall be transformational for our science. In the past successful projects have opened new parameter space and created the environment for new discoveries mostly by exploring new wavelength or resolution regimes. The ELTs do so not by opening new wavelength regimes but by dramatically changing the sensitivity through the use of adaptive optics routinely and for all astronomers. The optical and near infrared sky glows thereby creating a background that limits the sensitivity of observations. To beat the background a smaller projection on the sky of individual detecting elements in the instruments is needed. To match these smaller pixels to the sky background the sources must be made appropriately smaller as well. With the use of adaptive optics the blurring effects of the atmosphere can be compensated for and images at the diffraction limit of the telescope can be achieved.

With the benefit of wide field adaptive optics, now being demonstrated on 8-m telescopes [4], ELTs will be able to deliver 2 to 3 milli-arcsecond resolution over fields of many arcminutes. The technology, see below, is now in place to build the telescopes that our science requires.

\section{The observatory}

All three ELTs currently being designed are fully steerable segmented primary mirror telescopes enclosed in a dome for protection from the elements and the wind. In the sections below some aspects of the designs shall be described in more detail with particular focus on the European Extremely Large telescope. 


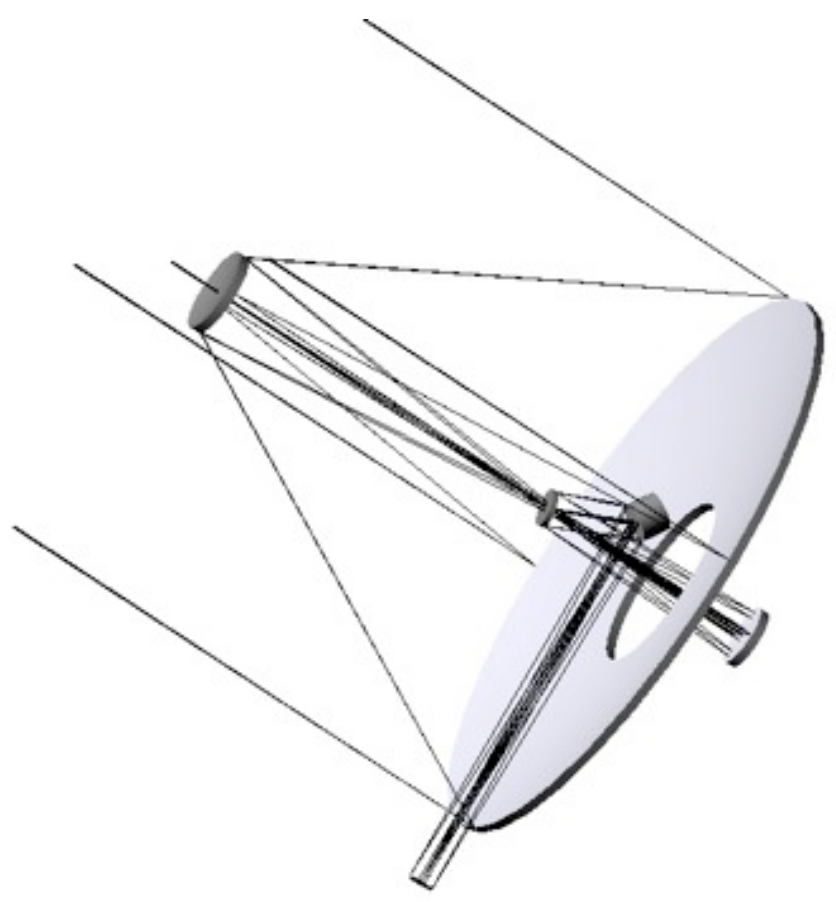

The optical design of the EELT is a three mirror on-axis anastigmat with two additional folding flat mirrors to steer the beam to the Nasmyth foci. With three powered surfaces, a 42-m $\mathrm{f} / 1$ primary, a convex 6-m secondary mirror and a 4.2-m tertiary mirror the EELT delivers unsurpassed image quality across its entire 10-arcminute field of view. Moreover, the beam is concentric to the pupil at all locations of the almost flat focal plane. This is a boon to instrumentation relaxing dramatically alignment tolerances. The two folding flats are sized at 2.5 and 2.7-m and have adaptive and field stabilization roles in the optical train of the telescope. The EELT is an adaptive telescope by inception and design.

With adaptive optics embedded in the telescope we have moved to the next natural step for optical instruments. With the NTT ESO pioneered the active telescope concept that allowed the 8-m class telescopes to be built and operated with excellent optical performance. Adaptive optics has reached a level of maturity that allows the next natural step to be taken. At Paranal today more than 7 adaptive optics systems some assisted by a Laser Guide star system are in routine turn-key operation without dedicated or expert support. The technology has now matured to the level that embedding it into a telescope is a natural path forwards and a technological enabler of new science. 


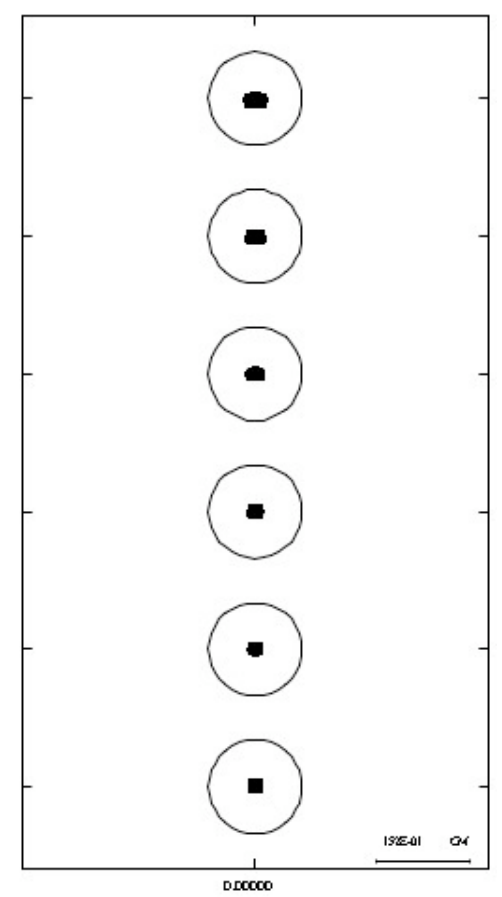

The image quality of the telescope from the centre field to the limit of 5 arc-minutes. The circle is 0.05 arc-seconds in size. The telescope is essentially diffraction limited at all positions of the focal plane.

\subsection{The mount}

All three telescope mounts are of the altitude azimuth type. One of the challenges for an ELT is to design a structure that will support the multiple segments of the primary mirror (984 in the case of the EELT) with small relative displacements (fractions of millimetres) as the telescope inclines from zenith to horizon. This is a problem solved for large radio telescopes but in those cases the altitude axis is below the primary mirror. For the EELT the altitude axis is above the primary making the provision of a massive structure to support the primary unfeasible for balancing reasons. Two industrial contractors have provided ESO with designs that meet the requirements for supporting the primary while at the same time having eigen frequencies that will allow for basic rejection of wind disturbances. A rocking chair mount is selected with two or more cradles. In the current baseline both the azimuth and altitude bearings are of hydrostatic type although bogies may be used instead. Direct drives as employed at the VLT are also considered a viable option although rack-pinion drives are also being investigated. 


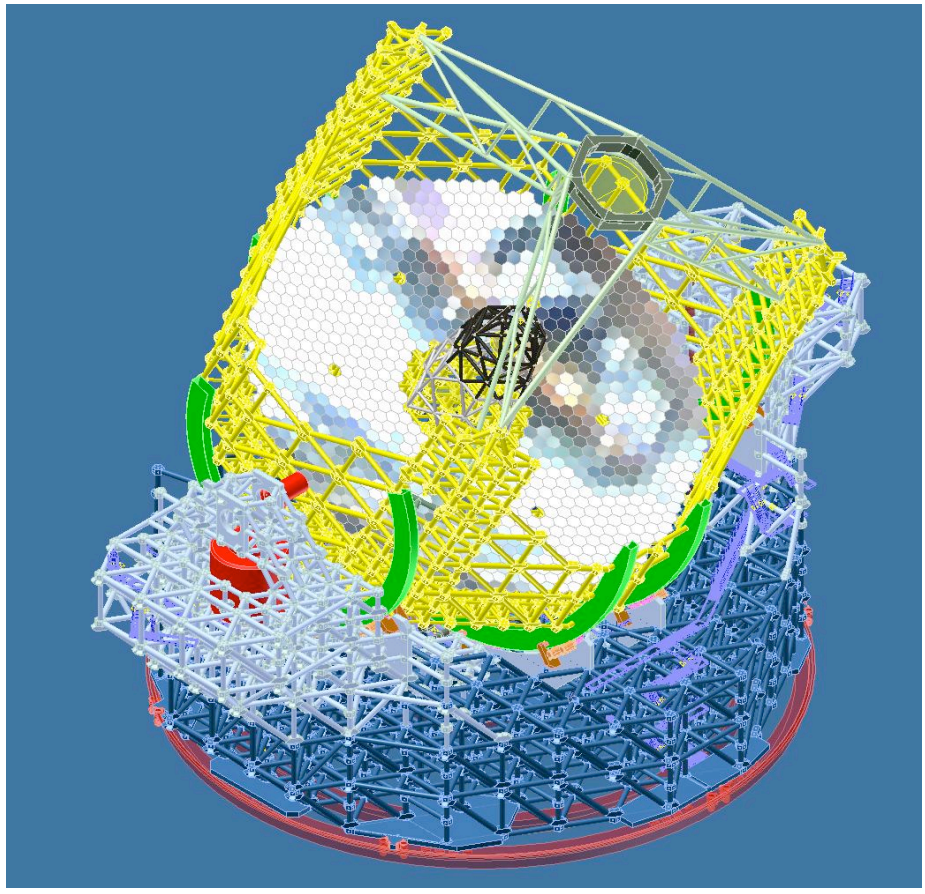

Example of telescope mount.

Particular attention is being paid to all the maintenance requirements for the telescope and the mirror cell. The active engagement of the ESO experience in construction and operation is being embedded into the project. In routine operations it is expected that one or more primary mirror segments would be exchanged per day for cleaning and recoating. For this purpose a sophisticated crane system is designed to be built into the telescope structure allowing easy and safe access to each component of the system.

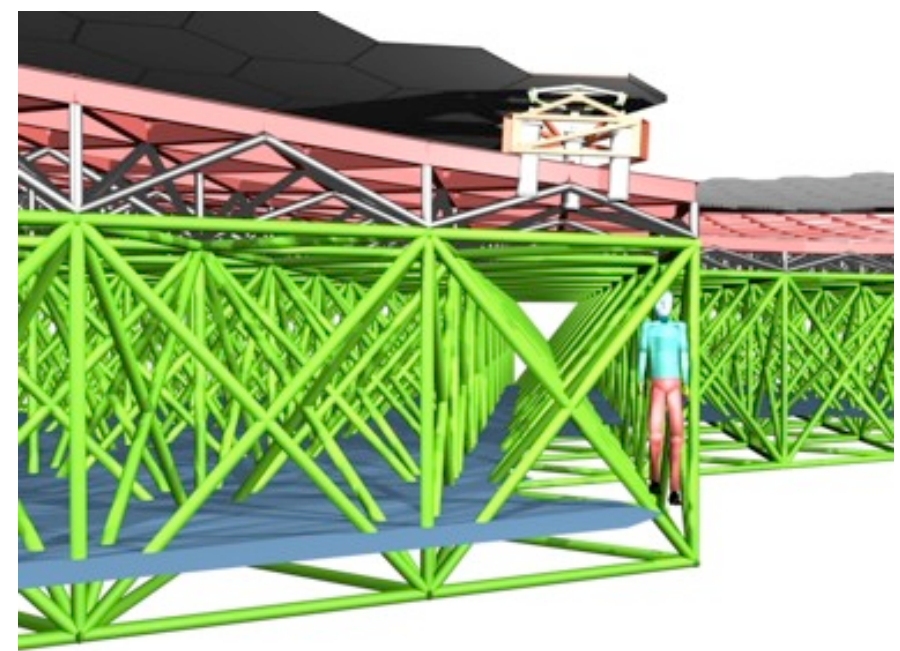

Accessibility of the back of the primary mirror through the framework structure. 


\subsection{Dome}

The telescopes are enclosed in massive domes to protect them from the elements during the day and to maintain the mirror surface clean. Three different options are considered by the projects for the enclosures. GMT is designing a carousel like "boxy" enclosure, TMT is using a semi-spherical carlotte design while the EELT is investigating the dome like enclosures with massive doors.

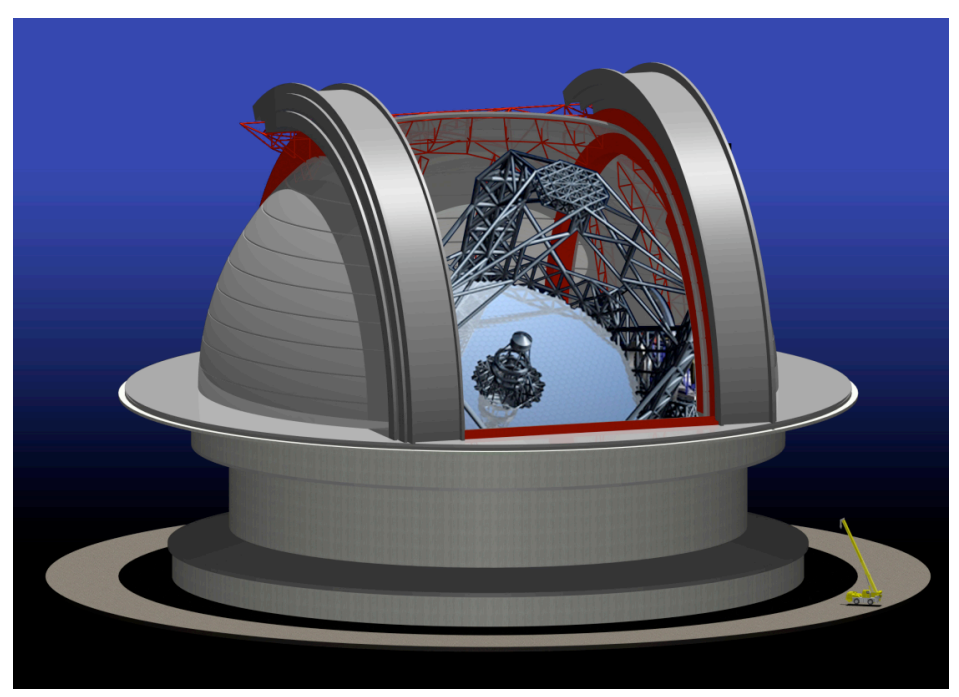

One example of a dome for the EELT.

For the EELT, two industrial firms have established preliminary designs for the dome, while two further contracts have been elaborated upon through the FP6 ELT design study activities on box-like and dome like enclosures. Wind tunnel testing is due to start in 2008. The dome of the EELT not only provides protection from the elements but critically ensures excellent ventilation for the telescope during observation periods.

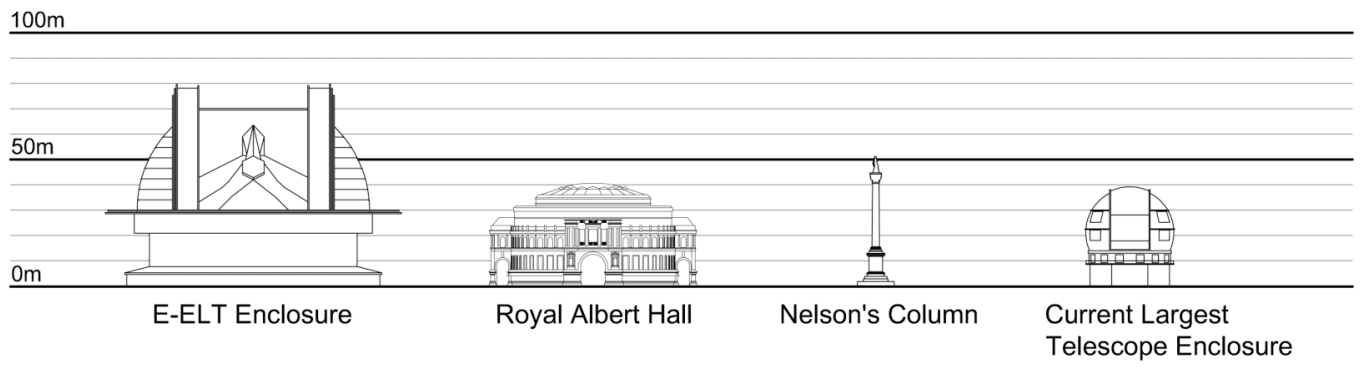

Comparison of the EELT enclosure with prominent structures.

The dome also provides for the air-conditioning of the telescope during the day in preparation for the night time observing. Detailed analysis has been undertaken to provide sufficient cooling to allow the telescope to match the external temperature at the beginning of the coming night. The dome also provides for the maintenance platforms that allow the 
exchange of the instruments at the Nasmyth foci and the access to the secondary mirror unit for maintenance at a horizon pointing position. The analysis of the dome has included CFD simulations and wind tunnel testing is planned for 2008.

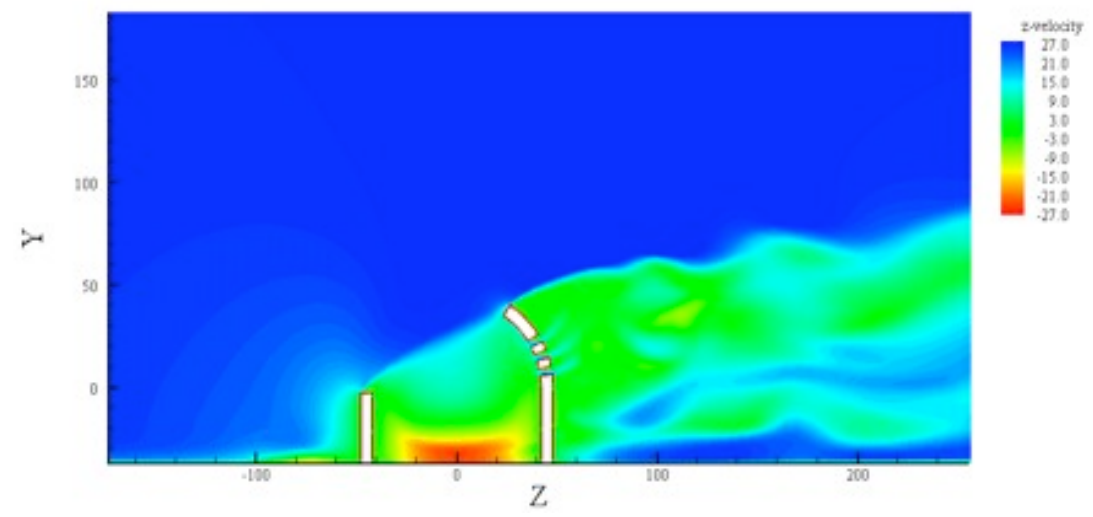

CFD analysis of the dome under $27 \mathrm{~m} / \mathrm{s}$ wind.

\subsection{Optics}

As mentioned above the EELT 42-m diameter primary is segmented with $9841.45-\mathrm{m}$ (point to point) hexagonal segments. For the EELT two industrial firms are currently producing a number of prototype segments of the exact prescription that the project will require in the final mass production. The hexagonal segments are to be made of glass or glass ceramic material, although silicon carbide is not excluded. A further industrial contract is designing and costing the production of the primary mirror cells. Solutions with 27 point and 18 point wiffle trees are being considered.

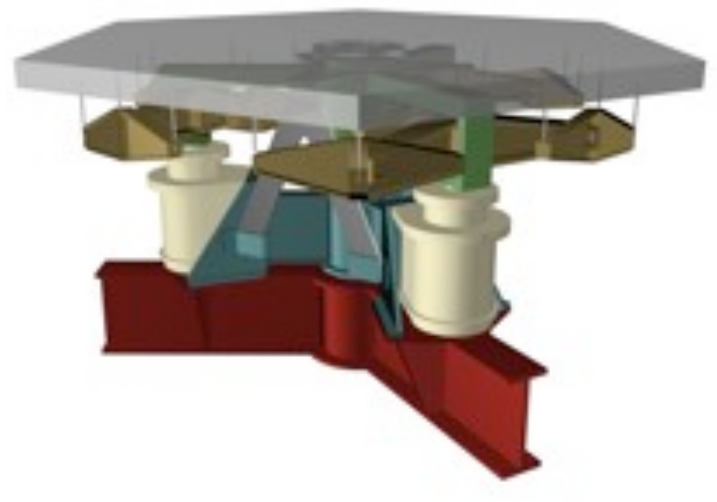

Concept for the mirror cell supporting each of the primary mirror segments 
The 6-m diameter and $100-\mathrm{mm}$ thin secondary mirror is significantly smaller than the $8-\mathrm{m}$ blanks made for the VLT and Gemini telescopes so there is confidence in the industrial partners in the production of the blank. The polishing of the mirror that is convex is more challenging but sub-aperture stitching techniques have already been used for the VLT secondary mirrors. Industrial contracts are being prepared for the preliminary design of the secondary mirror unit.

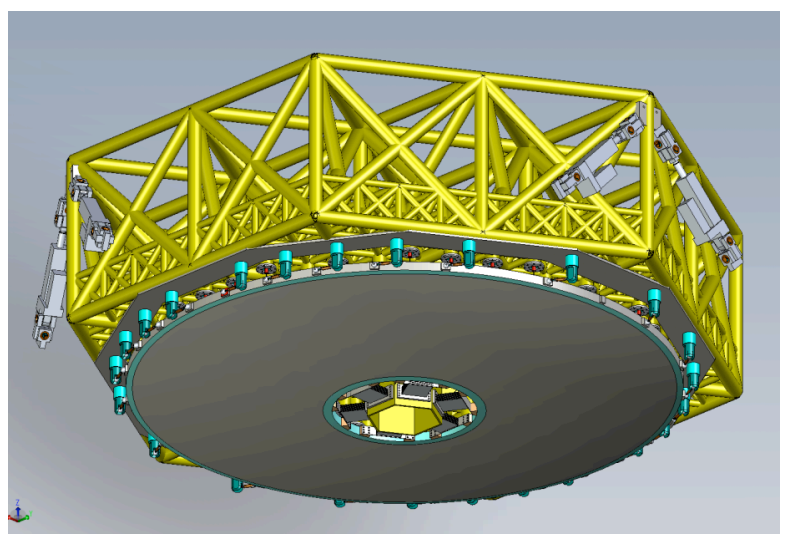

Conceptual design of the secondary mirror and its cell

The tertiary mirror is mildly aspheric and with a diameter of just over 4 metres, neither it nor its cell pose a particular challenge.

The quaternary and the fifth mirrors in the optical train form part of the adaptive optics train. Two industrial firms are engaged in a preliminary design of the quaternary mirror unit. The mirror will have over 5000 actuators and will provide ground layer correction for all instruments.

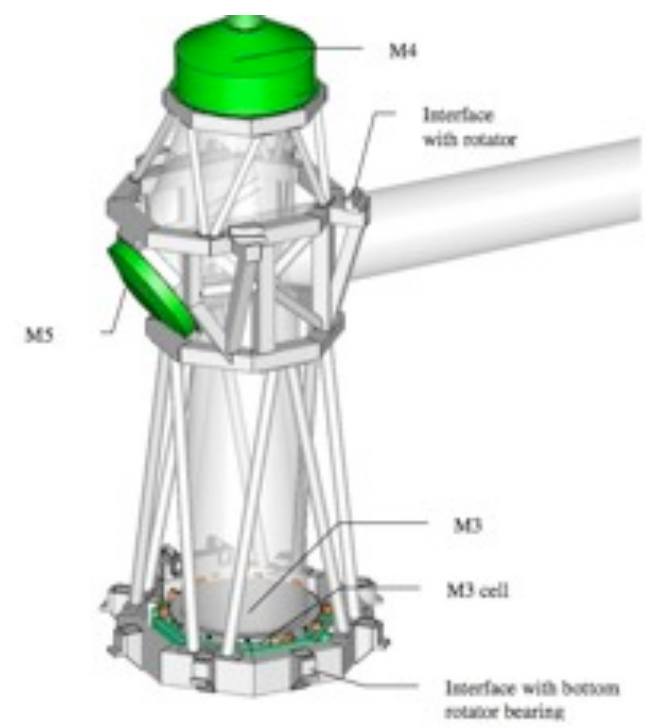

The Adaptive Relay Unit housed in the centre of the telescope 
The fifth mirror is a tip-tilt mirror and needs to be light-weighted. An industrial study into the design and construction of the mirror support unit has already been initiated. The production of the mirror itself has been studied by one industrial partner already and forms part of the design contract for the mirror support. Options to procure this mirror in $\mathrm{SiC}$ are also being explored.

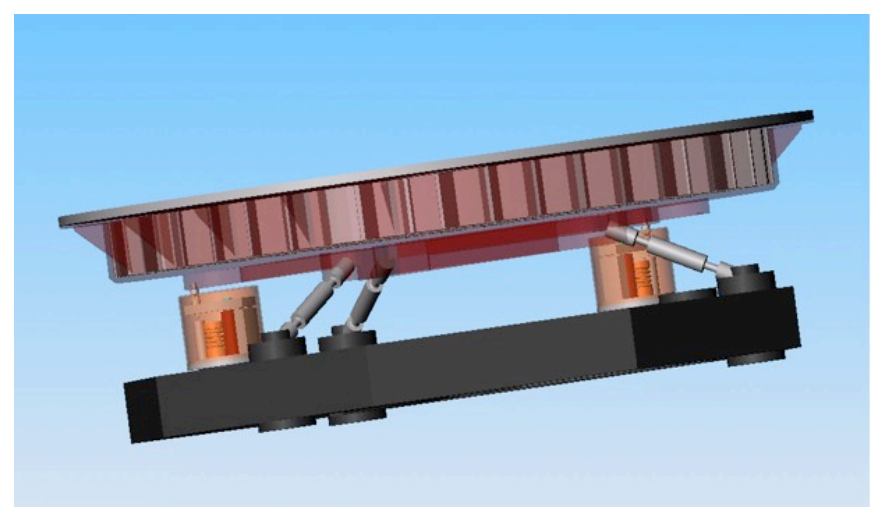

Conceptual design for the fifth mirror and its tip-tilt unit.

\subsection{Focal stations}

The telescope provides two Nasmyth focal stations and a coude focus for large instrumentation. At one Nasmyth focus a gravity invariant location is provided for though a folding mirror. The first or intermediate focus is also in principle available for instrumentation although it does not take advantage of the built in adaptive optics functions. The telescope provides sufficient space on the Nasmyth platforms to host multiple instruments and provide for a rapid exchange of the instrument receiving the telescope beam. 

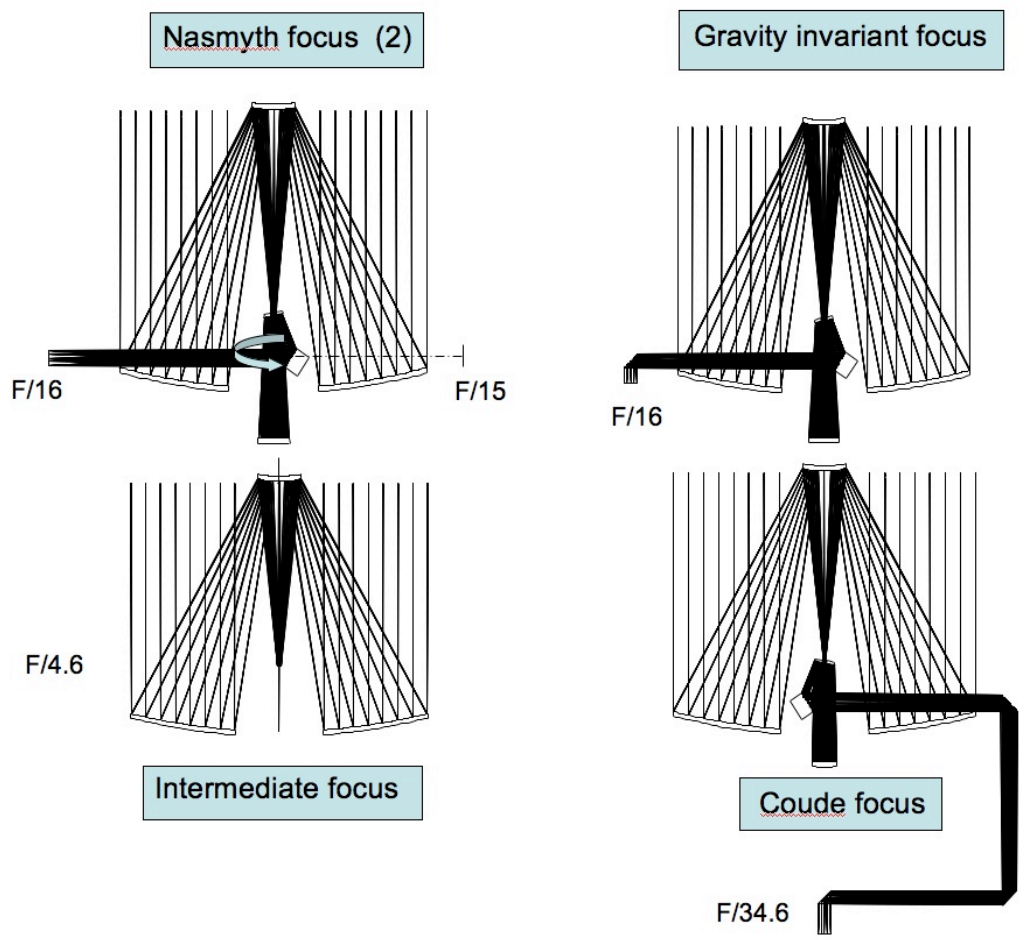

The different focal stations of the telescope

\subsection{Instrumentation}

A process of phase A design is underway for a number of instrument concepts. The instrumentation complement has been matched to the science cases. Under study are high spectral resolution ultra stable optical spectrographs, extreme adaptive optics instruments, multi-object spectrographs, multi-conjugate adaptive optics instruments and others.

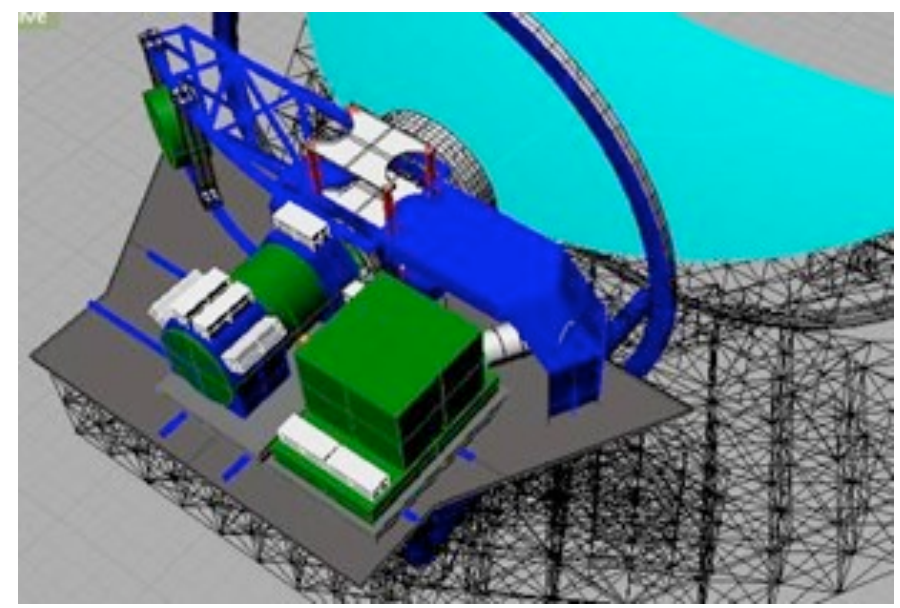

Distribution of instruments, including the MCAO module, on one Nasmyth platform 


\subsection{System engineering and performance analysis}

The performance of the telescope is being analyzed in great detail through multiple channels. As discussed earlier the design reference missions are establishing the science basis for the project. Within the engineering process a contract is in place with the university of Lund to simulate the EELT with their sophisticated end-to-end integrated model system. In parallel detailed analysis is ongoing within ESO. Within these activities a detailed Finite Element Analysis and control simulation of the telescope has been made to derive requirements on the enclosure and telescope mechanics as well as the inputs for the design of the deformable mirror and the tip-tilt stage. The adaptive optics performance of the telescope is being simulated in the various operational modes and Ground Layer Adaptive Optics is shown to provide wide field seeing improvement comparable to that achieved at Paranal with the Multiconjugate Adaptive Optics Demonstrator during 2007. With GLAO, LTAO, MCAO and MOAO (acronyms that the reader is encouraged to discover) all planned to be used with the EELT these simulations are critical components of the design process.

The mount performance is already at a very respectable $2.3 \mathrm{~Hz}$ locked rotor eigen frequency and the Ground Layer Adaptive optics performance of the system is achieved even at relatively high wind loading conditions of $10 \mathrm{~m} / \mathrm{s}$.

Significant attention is being paid to the earthquake analysis of the system. As with the VLT a particularly conservative approach is being taken for the size of earthquake that the telescope is to be able to survive.

The system engineering process uses DOORS to manage the requirements and SYSML for analysis of complex control issues.

\subsection{Infrastructure \& site}

A number of potential sites are being investigated for the EELT, including (alphabetically) locations in Argentina, Canaries, Chile, Morocco and Mexico. The site characterisation process is expected to last until late 2008 or early 2009. In the mean time the project is making assumptions for the infrastructure needed on the basis of the ESO experience opening the Paranal observatory in Chile. 


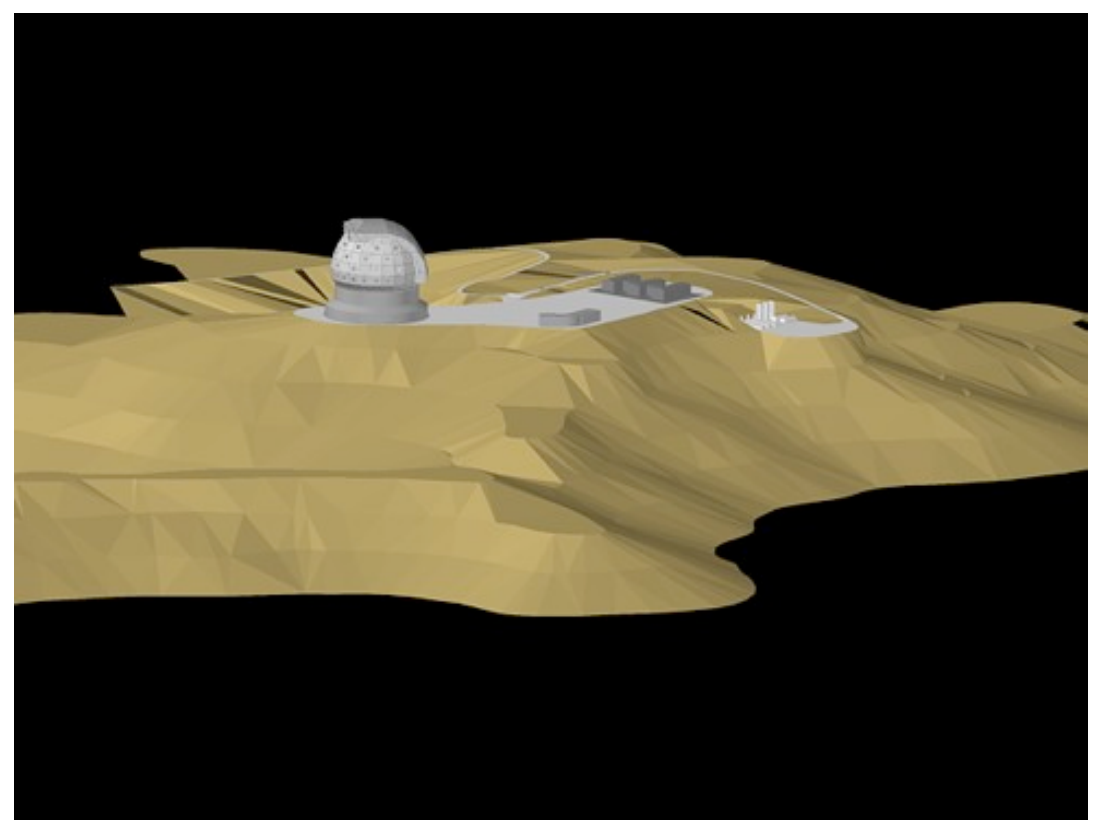

Conceptual design of the observatory site. Note this is not a real site.

\section{4. $\quad$ Schedule \& Budget}

The EELT phase B activities are expected to conclude in late 2009 or early 2010 with a construction proposal. Construction time for the complete facility is expected to last for 7 years dictated mostly by the serial production of the primary mirror segments. The preliminary cost for the project at the time of the start of the phase B was 900 million Euro including instrumentation and the first year of operation of the facility.

\section{External activities}

In addition to the EELT Phase B activities in ESO, a significant FP6 design study project has been underway since 2005 with industrial and academic participation from a large number of partners. Advanced Phasing experiments, new dome designs, bogie test beds, wind evaluation breadboards, new edge sensor technologies, powerful actuators for the mirror segments, site testing, scientific operations and other activities are all being investigated in this collaboration. Within the FP7 activities ESO has been successful in creating another collaboration to complement the internal activities in system engineering, industrial relations, instrumentation etc. 


\section{Conclusion}

The European Extremely Large Telescope programme is in phase B and ESO hopes to start construction in 2010. The telescope will not only open new vistas to our universe with unparalleled capabilities in adaptive optics and instrumentation but also powerfully complement the exciting astronomy expected to come from ALMA.

\section{References}

[1] http://www.tmt.org/foundation-docs/TMT-DSC-2007-R1.pdf

[2] http://www.gmto.org/sciencecase/GMT-ID-01404-GMT_Science_Case.pdf

[3] http://www-astro.physics.ox.ac.uk/ imh/ELT/Book/ELT_brochure_2.pdf

[4] http://www.eso.org/public/outreach/press-rel/pr-2007/pr-19-07.html 